\title{
CORRECTIONS
}

\section{The crisis in diabetes care in England}

There are a few errors in this Editorial (BMJ 2012;345:e5446, doi:10.1136/bmj.e5446) by Gerry Rayman and Anne Kilvert. The first error is in the first line of the second paragraph: "The National Diabetes Audit reported that in 2010-11 only half of people with diabetes received all of the recommended nine care processes..." In fact, the National Diabetes Audit that is being referenced here was published in 2011, but related findings from the period of 2009-10.

The second line of this second paragraph also incorrectly asserts that "death in young women (aged 15-34 years) with type 1 diabetes has increased ninefold since the 2007-08 audit."

However, the ninefold increase was not in relation to the 2007-8 audit, but represented the difference in the death rate between young women with diabetes and young women without diabetes.
The second sentence should have reflected this by reading: "An estimated 24000 diabetes related deaths each year may have been preventable, and the rate of death in young women (aged 15-34 years) with type 1 diabetes was ninefold higher than the rate in non-diabetic young women."

There is also an error in the fourth paragraph. The sixth sentence states that "the NAO found that only $£ 2 \mathrm{~m}(0.0005 \%)$ of the money spent on diabetes is allocated to patient education." The percentage cited has been calculated incorrectly and should have been $0.05 \%$ [not " $0.0005 \%$ "].

Cite this as: BMJ 2012;345:e5565

(๑) BMJ Publishing Group Ltd 2012 